\title{
The ESSnuSB/HIFI Design Study
}

\section{Loris D'Alessi ${ }^{a, *}$ and Tord Ekelöf ${ }^{b}$ for the ESS $v$ SB Collaboration}

\author{
${ }^{a} I P H C$, Université de Strasbourg, CNRS/IN2P3 \\ F-67037 Strasbourg, France \\ ${ }^{b}$ Uppsala University, \\ P.O. Box 256, 75105 Uppsala, Sweden \\ E-mail: loris.dalessi@iphc.cnrs.fr, tord.ekelof@physics.uu.se
}

The ESS 5 MW linac will be the world's most powerful accelerator, enabling with its $10^{16} 2$ $\mathrm{GeV}$ protons per second the production of the world's most intense flux, not only of neutrons, but also of neutrinos and muons. This opens unique opportunities for High Intensity Frontier fundamental physics. The EU supported Design Study of an ESS neutrino Super Beam (ESS $v$ SB) for long baseline neutrino oscillation measurements, based on an upgrade of the ESS facility, has been under way since 2018 with the participation of physicists from fifteen European institutions. In this study is designed the upgrade of the facility in order to increase the linac power to 10 MW by the provision of extra $\mathrm{H}^{-}$pulses between the proton linac pulses and provide a ca $400 \mathrm{~m}$ circumference accumulator ring to compress the $3 \mathrm{~ms}$ long linac pulses to $1.3 \mu \mathrm{s}$, a set of four highpower neutrino targets with focusing horns and a kiloton near and a megaton far water Cherenkov neutrino detector, the latter at a location of $360 \mathrm{~km}$, alternatively $540 \mathrm{~km}$, from ESS, both of which are near the location of the second neutrino oscillation maximum. The time of the publication of the ESSnuSB Design Study report is approaching and highlights among achieved design results are presented. Recently a study of the use of the intense muon flux produced concurrently with neutrinos has been started, aiming at a design of, in the first stage, a nuSTORM low-energy facility and a Proton Complex for production of $2 \mathrm{~ns} 10^{15}$ proton bunches for a future Muon Collider. The plan for this High Intensity Frontier Initiative (HIFI) design work is presented.

\footnotetext{
*** The European Physical Society Conference on High Energy Physics (EPS-HEP2021), ***

*** 26-30 July 2021 ***

*** Online conference, jointly organized by Universität Hamburg and the research center DESY ***
}

\footnotetext{
${ }^{*}$ Speaker
} 


\section{Introduction}

Current long baseline neutrino oscillation experiments are designed for the precise measurement of the neutrino oscillation parameters, which in the three-flavour scenario describe the neutrino flavour oscillations. Those of these parameters that remain to be measured with precision are: the neutrino mass hierarchy $\Delta m_{31}^{2}$, the octant of the mixing angle $\theta_{23}$ and the CP-violating phase $\delta_{C P}$. The value of the latter parameter can, in particular, provide an explanation for the matter-antimatter asymmetry observed in the Universe. The T2K [1] experiment provides a best-fit value for $\delta_{C P}$ that is close to $-90^{\circ}$, therefore maximal CP violation, whereas the data analysis results from the $\mathrm{NO} v \mathrm{~A}$ experiment [2] do not show a preference for $\mathrm{CP}$ conservation versus violation. For the two experiments taken together, the values $\delta_{C P}=0^{\circ}$ and $-90^{\circ}$ are allowed within the $3 \sigma$ C.L. . More data will thus be required to determine the most likely value. In the quest for higher precision in the measurement of $\delta_{C P}$, new long baseline neutrino oscillation experiments have been proposed, such as in Japan the T2HK experiment [3] and in the US DUNE [4].

In Europe, the study for a European neutrino Super Beam started with the EUROnu Design Study [5], which ran from 2008 to 2012 and was supported by the FP7 European Research Program. The measurement, in 2012, of a relatively large $\theta_{13}$ implied that the sensitivity of the CP violation measurement is close to three time larger at the second neutrino oscillation maximum as compared to at the first maximum. The ESS $v$ SB Design Study [6], which builds in part on the Design Study results of EUROnu, is proposing to use the ESS linac, currently under construction in Lund (Sweden) [7], as proton driver. The ESS $v$ SB project is funded by the EU Horizon 2020 program for 4 years, from 2018 to 2021, and at the end of the design study a Conceptual Design Report (CDR) will be delivered.

\section{Overview of the ESSnuSB Experiment}

The European Spallation Source will be the world's highest brightness neutron source. Neutrons will be produced in a dedicated target facility, using protons accelerated in a superconducting linac. In its fully operational phase, the ESS linac will produce proton pulses of $5 \mathrm{MW}$ average power, $2 \mathrm{GeV}$ proton kinetic energy, $2.86 \mathrm{~ms}$ pulse duration and $14 \mathrm{~Hz}$ repetition rate. The duty cycle of the machine will be, therefore, only $4 \%$.

The ESS $v$ SB project proposes to double the duty cycle of the ESS linac in order to obtain a separate proton pulse to be delivered to a dedicated target station for the production of the neutrino beam. The neutrinos are produced from the decay of mesons, mainly pions, produced in the interaction of the protons with a solid target and focused by a MiniBooNE-like [8] horn.

Because of its high intensity, the beam needs to be split in four beams to be sent to 4 target/horn assemblies, each target interacting with a $1.25 \mathrm{MW}$ beam. Since the pulsed magnetic field in the horn will be produced by $350 \mathrm{kA}$ current pulses, which need to be kept as short as possible to limit the high heat dissipation, the proton pulse duration needs to be reduced to the order of the $\mu$ s level. The $\mu$ s pulse duration is also required to reduce the contamination in the neutrino detection window due to the atmospheric neutrinos. In order to achieve the $\mu$ s pulse duration, the proton pulse will be injected in an accumulator ring. 


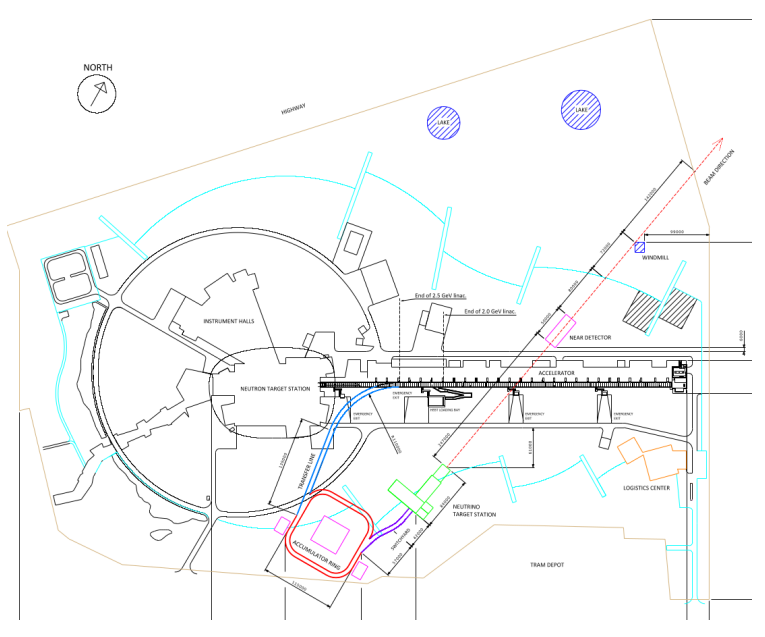

Figure 1: Layout of the ESS $v$ SB accumulator ring, target station and near detector on the ESS site.

The layout of the proposed experiment on the ESS site, with the accumulator ring, the target station and the near detector location, is shown in Figure 1. In the next sections the main characteristics of the experiment will be described in more details.

\subsection{The ESS Linac Upgrade}

For the production of the neutrino beam, it is proposed to inject in the linac a beam of $\mathrm{H}^{-}$ ions, rather than a proton beam, and to accelerate the ions up to $2.5 \mathrm{GeV}$ before the injection in the accumulator ring. The use of $\mathrm{H}^{-}$ions that will be stripped of their two electrons at the entrance of the accumulator ring is required in order to enable the addition of more protons to the already circulating proton beam during the pulse. The increase to $2.5 \mathrm{GeV}$ kinetic energy will reduce the space charge effects as well as the required average beam current. One option when adding the $\mathrm{H}^{-}$ ion source is to merge the proton and $\mathrm{H}^{-}$beams in the LEBT and use the same RFQ and the other option is to use two separate front-end sections and merge the beam in the MEBT [9].

\subsection{The Accumulator Ring}

In its current design, the accumulator ring has a circumference of $384 \mathrm{~m}$. It will consists of a lattice divided in four arcs and four straight sections [10]. The $\mathrm{H}^{-}$ion beam, divided in 4 batches with a separation gap of $0.1 \mathrm{~ms}$, will be injected in the accumulator ring through multiturn injection. The stripping of the ion electrons will be made with the use of graphite foils of $\mu \mathrm{m}$ thickness. The use of laser-assisted stripping represents an alternative option that will be investigated more in detail in the future. Anticorrelated painting technique allows to obtain circular uniform proton beam profile. The layout of the Accumulator Ring is shown on the left panel of Figure 2.

\subsection{The Target Station}

The target station will consists of the 4 target-horn systems, the decay tunnel and the beam dump. The layout of the target station is shown in the central panel of Figure 2. Each of the four targets consists of a packed bed of $1.5 \mathrm{~mm}$ radius titanium spheres, contained in a $1.5 \mathrm{~cm}$ radius and $78 \mathrm{~cm}$ length titanium canister and cooled using forced helium gas flow. The shape of the horn and 

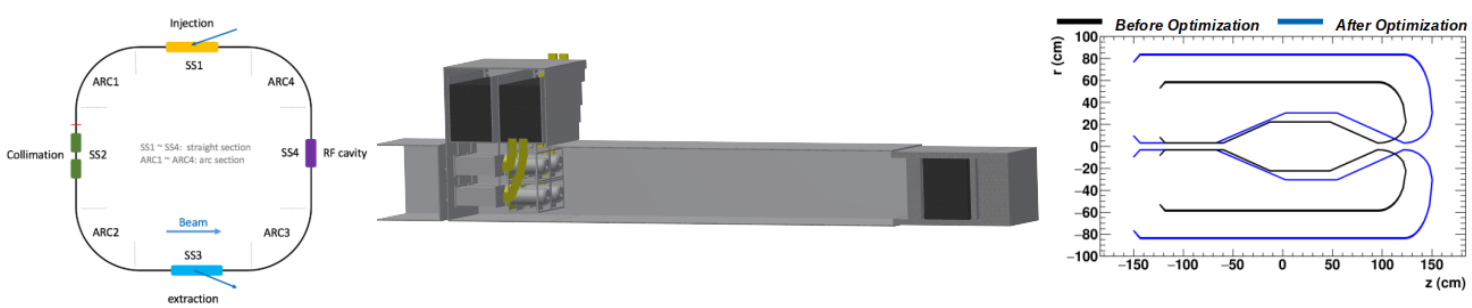

Figure 2: Layout of the accumulator ring (left), target station (center) and horn profile before and after optimization (right).

decay tunnel are tuned to optimize the neutrino beam quality. The thermal and mechanical stresses on the components of the target station, in particular the target, horn and beam dump, are currently under investigation. Current optimization studies, based on Genetic Algorithm calculations, show that a larger size for the horn and the tunnel, compared with the proposed $2.5 \mathrm{~m}$ length, $60 \mathrm{~cm}$ radius horn and $25 \mathrm{~m}$ decay tunnel length, result in a significantly increase in the number of detected neutrinos [11]. Both the old horn design and the new optimized one are shown in the right panel of Figure 2.

\subsection{The Near and Far Detectors}

The far detector will consist of two identical modules, having a total fiducial volume of 538 kt and equipped with about $10^{5} 20$ " PMTs to provide $40 \%$ optical coverage. It will be located near the second oscillation maximum, where the sensitivity to the CP violation effect is expected to be near to 3 times larger than at the first oscillation maximum. Currently, the Garpenberg mine, $540 \mathrm{~km}$ distance from ESS, and the Zinkgruvan mine, at $360 \mathrm{~km}$ distance, are being considered as alternative locations for the far detector location. The near detector consists of a 1 kton water Cherenkov detector, complemented with a magnetized Super Fine Grained Detector (SFGD) and an emulsion setup, the latter similar to that of the NINJA experiment [12].

\subsection{Physics Performance of the Experiment}

The recent optimization of the horn and decay tunnel geometry, by means of the Genetic
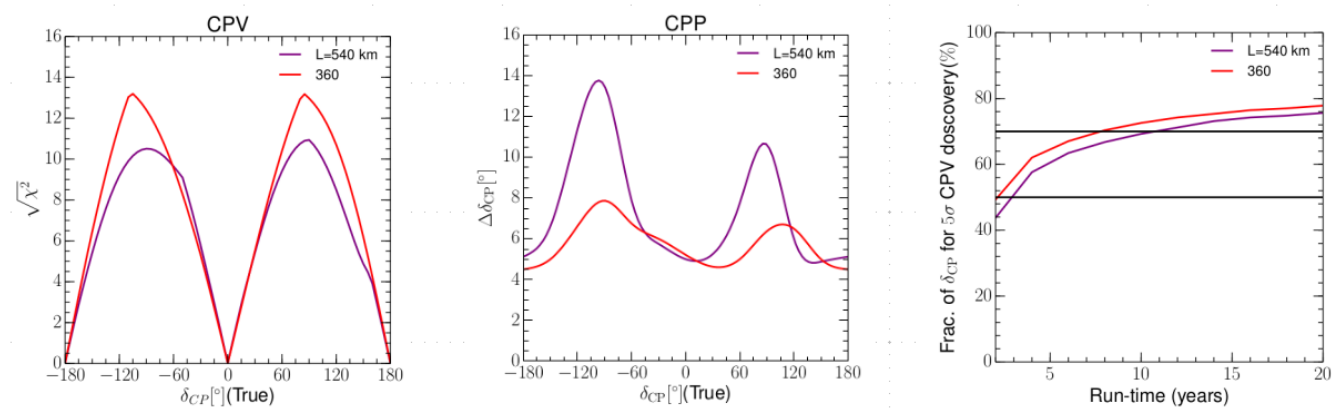

Figure 3: Reach of the $\mathrm{ESS} v \mathrm{SB}$ experiment for the CP-violating phase discovery (left), precision achievable on the measurement of $\delta_{C P}$ (center), both as function of $\delta_{C P}$, and fraction of $\delta_{C P}$ values as function of the exposure time (right) for the location of Garpenberg $(540 \mathrm{~km})$ and Zinkgrouvan $(360 \mathrm{~km})$. 

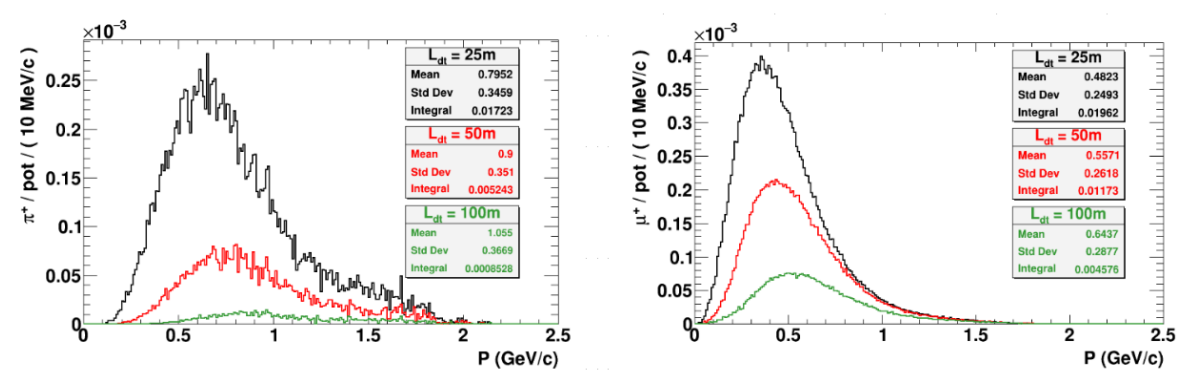

Figure 4: Distribution of pions (left) and muons (right) as function of the decay tunnel length.

Algorithm and improved efficiency in the neutrino energy reconstruction, has resulted in a significant increase in the discovery reach for CP violation and the precision with which the $\delta_{C P}$ will be measured, corresponding to an uncertainty of not more than $8^{\circ}$ for all values of $\delta_{C P}$, as shown in Figure 3 [13].

\section{The HIFI Initiative}

A high flux of muons will be produced concurrently with the neutrino production. Figure 4 shows the pion and muon flux at the beam dump level for different lengths of the decay tunnel. The uniquely high flux of muons that can be generated at ESS, together with the equally high intensity flux of electron and muon neutrinos from their decay, has led the $\operatorname{ESS} v \mathrm{SB}$ collaboration to enlarge the scope of its design study with what has been named the "High Intensity Frontier Initiative", aiming at exploiting the further potential of the ESS high power accelerator for Particle Physics [14]. The enlarged scope includes design studies of a Low Energy nuSTORM facility for high precision neutrino cross-section measurements and of a Proton Complex Test Facility, to produce 2 ns long bunches of $10^{15}$ protons, as required for a Muon Collider. The feasibility of the latter will be explored within the programme of the International Muon Collider Collaboration (IMCC) [15]. The latter study could also represent a first step towards a design study of a Low Energy Muon Collider Higgs Factory located at the ESS site [16].

\section{Conclusions}

The ESS $v$ SB design study has demonstrated the potentiality of the ESS site to host a worlduniquely intense neutrino Super Beam in Europe, with which the leptonic CP violating phase $\delta_{C P}$ will be measured with unprecedented precision. The scope of the ESS $v$ SB design Study has recently been widened to encompass a design study of a low energy nuSTORM facility for neutrino cross-section measurements and that of a Proton Complex Test Facility as part of the International Muon Collider design study.

\section{Acknowledgments}

This project has received funding from the European Union's Horizon 2020 research and innovation programme under grant agreement No 777419. This work has been in part funded 
by the Deutsche Forschungsgemeinschaft (DFG, German Research Foundation) - Projektnummer 423761110. This work has been in part funded by Ministry of Science and Education of Republic of Croatia grant No. KK.01.1.1.01.0001.

\section{References}

[1] T2K Collaboration, K. Abe et al., Constraint oon the matter-antimatter symmetry-violating phase in neutrino oscillations, Nature 580, no. 7803 339-344 (2020) [arXiv:1910.03887] [Erratum: Nature 583, E16 (2020)].

[2] NOvA Collaboration, M. A. Acero et al., An Improved Measurement of Neutrino Oscillation Parameters by the NOvA Experiment, [arXiv:2108.08219 [hep-ex]].

[3] Hyper-Kamiokande Collaboration, K. Abe et al., Physics potentials with the second HyperKamiokande detector in Korea, PTEP 2018, no. 6063 C01 (2018), [arXiv:1611.06118].

[4] DUNE Collaboration, B. Abi et al., Deep Underground Neutrino Experiment (DUNE) Far Detector Technical Design Report, Volume II: DUNE Physics, arXiv:2002.03005.

[5] T. R. Edgecock et al., High intensity neutrino oscillation facilities in Europe, Phys. Rev. ST Accel. Beams 16, 021002 (2013) [arXiv:1305.4067 [physics.acc-ph]].

[6] E. Baussan et al., A very intense neutrino super beam experiment for leptonic CP violation discovery based on the European spallation source linac, Nucl. Phys. B 885, 127-149 (2014).

[7] S. Peggs et al., "Technical Design Report ESS”, 2013 eval.esss.lu.se/cgi-bin/public/DocDB/ShowDocument?docid=274

[8] A. A. Aguilar-Arevalo et al., Neutrino flux prediction at MiniBooNE, Phys. Rev. D79, 072002 (2009).

[9] B. Gålnander et al., Status of ESS linac upgrade studies for ESSnuSB, in: 10th International Particle Accelerator Conference, 2019, p. MOPTS082.

[10] Y. Zou, The accumulator ring for the ESSnuSB project - a progress report, PoS NuFact2019 (2019) 061.

[11] L. D'Alessi et al., "Optimization of the Target Station for the ESSnSB Project Using the Genetic Algorithm", NeuTel Conference 2021.

[12] A. Hiramoto et al., Phys. Rev. D 102, 072006 (2020) [arXiv:2008.03895]

[13] ESS $v$ SB Collaboration, A. Alekou et al. [arXiv:2107.07585 [hep-ex]]

[14] HIFI workshop event web site: indico.cern.ch/event/849674/

[15] IMCC web site: https://muoncollider.web.cern.ch/ .

[16] C. Rubbia, arXiv:1908.05664v3 [physics.acc-ph] (2019). 